\title{
GERMINAÇÃO DE SEMENTES DE PALMEIRA-RÁFIA: EFEITO DE TRATAMENTOS PRÉ-GERMINATIVOS ${ }^{1}$
}

\author{
Petterson Baptista da Luz², Armando Reis Tavares ${ }^{3}$, Patrícia Duarte de Oliveira Paiva ${ }^{4}$, Francismar \\ Francisco Alves Aguiar ${ }^{3}$ e Shoey Kanashiro ${ }^{3}$
}

\begin{abstract}
RESUMO - A propagação da palmeira-ráfia (Rhapis excelsa), palmeira ornamental de grande valor comercial, é realizada através de sementes ou divisão de touceiras. Entretanto, a germinação das sementes não é uniforme, e o crescimento da planta é considerado lento. Neste trabalho, objetivou-se comparar a utilização de tratamentos pré-germinativos para acelerar e uniformizar a germinação de sementes de R. excelsa. Avaliou-se o efeito das escarificações mecânica (lixar um lado ou dois lados da semente), térmica (imersão em água a $100^{\circ} \mathrm{C}$ durante 1, 2 ou 4 min) e química (imersão em ácido sulfúrico $98 \%$, durante 1, 2 ou 4 min), bem como a sua embebição em soluções contendo BAP (benzilaminopurina) nas concentrações de $0,25,50$ ou $100 \mathrm{mg} \mathrm{L}^{-1}$ e GA 3 (ácido giberélico) nas concentrações de 0, 100, 200 ou $300 \mathrm{mg} \mathrm{L}^{-1}$ na germinação e velocidade de germinação de sementes da espécie. Os resultados evidenciaram que os tratamentos pré-germinativos utilizados não influenciaram a porcentagem de germinação nem o índice de velocidade de emergência das sementes.
\end{abstract}

Palavras-chave: Rhapis excelsa, Palmae e índice de velocidade germinação.

\section{LADY PALM SEED GERMINATION: EFFECTS OF PRE-GERMINATION TREATMENTS}

\begin{abstract}
Lady palm (Rhapis excelsa) is an ornamental plant with great commercial value, usually cultivated as vase plant. It is propagated by seed or clump division. Seed germination is not uniform and plant growth is slow, leading to delayed plant production. The research was carried out at the Federal University of Lavras and aimed to compare three scarification methods for a rapid and uniform germination of $\boldsymbol{R}$. excelsa seeds. The effect of mechanical scarification (with sandpaper in one or both sides of the seed), thermal scarification (immersion in $100^{\circ} \mathrm{C}$ water over 1, 2 or 4 minutes) and chemical scarification (immersion in $98 \%$ sulfuric, during 1, 2 or 4 minutes)was evaluated on seed germination, using the parameters germination percentage and the index of emergence speed (IES). The results showed that germination percentage and the index of emergence speed were not affected by seed scarification.
\end{abstract}

Keywords: Rhapis excelsa, Palmae and speed of germination Index.

\section{INTRODUÇÃO}

A palmeira Rhapis excelsa (Thunberg) Henry ex. Rehder é pertencente à família Palmae (Arecaceae), que compreende 198 gêneros e aproximadamente 2.650 espécies em todo o mundo (DOMINGUES, 1995). A espécie possui valor ornamental por ser pouco exigente em relação à luminosidade, tolerando para o seu desenvolvimento baixa luminosidade ou pleno sol quando adulta, podendo ser utilizada como planta de vaso.

As informações relacionadas ao período médio necessário para a germinação de sementes de $R$. excelsa são bastante divergentes, variando de 50 a 130 dias (McKAMEY, 1989; LORENZI et al., 2004). Segundo

\footnotetext{
${ }^{1}$ Recebido em 17.04.2007 e aceito para publicação em 22.08.2008.

${ }^{2}$ Departamento de Agronomia da Universidade do Estado de Mato Grosso-UNEMAT. E-mail: <petterbaptista@ yahoo.com.br>.

${ }^{3}$ Seção de Ornamentais do Instituto de Botância. E-mail: <atavares2005@yahoo.com.br>.

${ }^{4}$ Departamento de Agricultura da Universidade Federal de Lavras (UFLA). E-mail: <pdolivei@ufla.br>.
} 
Koebernick (1971), muitos fatores podem afetar a germinação de sementes de palmeira como espécie, temperatura, tipo de substrato, umidade e aeração do substrato e o período de armazenamento. Aguiar et al. (2005), estudando o efeito da luz, temperatura e substrato na germinação de $R$. excelsa, observaram que a temperatura de $25^{\circ} \mathrm{C}$ e o substrato areia aumentaram a porcentagem e o índice de velocidade de germinação da espécie, independentemente da presença ou ausência de luz.

A retirada da polpa (epicarpo e mesocarpo) é recomendada (MEEROW, 1991; BROSCHAT, 1994; LORENZI et al., 2004), visto que acelera a germinação de sementes de algumas espécies como Euterpe oleracea (BOVI e CARDOSO, 1976a), Euterpe edulis (BOVI e CARDOSO, 1976b) e Archontophoenix alexandrae (MAEDA et al., 1987), embora prejudicial no armazenamento de Euterpe espiritosantensis (MARTINS et al., 2000). Ferreira e Gentil (2006), estudando a emergência de sementes de tucumã (Astrocaryum aculeatum, Arecaeae), observaram que os fatores retirada do endocarpo e a embebição das sementes aumentaram a emergência. já Elias et al. (2006), pesquisando a mesma espécie, verificaram que a profundidade do poro germinativo no substrato proporcionou aumento da emergência e diminuição da porcentagem de sementes dormentes.

Figliolia et al. (1987), comparando a germinação de sementes de Euterpe edulis provenientes dos tratamentos frutos com polpa, despolpados após a imersão em água durante $24 \mathrm{~h}$ e escarificados mecanicamente, concluíram que as sementes, nos dois últimos tratamentos citados, germinaram de forma mais rápida e uniforme que as do tratamento-controle.

A ocorrência de dormência, que inibe a germinação de sementes mesmo em condições favoráveis (POPINIGIS, 1985; CARVALHO e NAKAGAWA, 1988), tem sido apontada como uma das principais causas de variação no período de germinação em palmeiras (MULLETT et al., 1981; VILLALOBOS et al., 1992ab).

Segundo Odetola (1987), não existe dormência em relação ao embrião, que se desenvolve continuamente após a maturação do fruto; porém, várias espécies da família Arecaceae apresentam dormência física em graus variados, demandando tratamentos como embebição em água ou em substâncias químicas reguladoras de crescimento, estratificação, escarificação química ou mecânica ou, mesmo, graus de exposição à luminosidade.
Viana (2003), estudando o efeito de quatro temperaturas (ambiente, $30{ }^{\circ} \mathrm{C}, 20-30{ }^{\circ} \mathrm{C}$ e $25-35^{\circ} \mathrm{C}$ ) com ou sem escarificação mecânica na germinação de sementes de Livistona rotundifolia, observou que os melhores resultados foram obtidos com escarificação sob temperatura alternada de $25-35^{\circ} \mathrm{C}$. Já Pivetta et al. (2005) verificaram que sementes de Syagrus schizophylla escarificadas mecanicamente expressaram maior porcentagem e germinação mais rápida quando comparadas com sementes não escarificadas.

Bovi e Cardoso (1976b) estudaram o efeito das escarificações mecânica (raspagem com agulha na região do poro vegetativo das sementes) e química (ácido sulfúrico por 5 ou $10 \mathrm{~min}$ ) na germinação de sementes de Euterpe edulis, concluindo que não houve diferença entre sementes escarificadas e não-escarificadas e que o ácido sulfúrico por período superior a $5 \mathrm{~min}$ foi prejudicial.

Bovi e Cardoso (1976a), estudando também a imersão em água corrente e água quente $\left( \pm 80^{\circ} \mathrm{C}\right)$ e escarificação química (ácido sulfúrico a $75 \%$ por 5 ou $10 \mathrm{~min}$ ) na germinação de sementes de Euterpe oleracea, concluíram que tanto o uso do ácido sulfúrico quanto o de água quente não foram satisfatórios.

Nagao et al. (1980) conseguiram acelerar a germinação de sementes de Archontophoenix alexandrae por meio de escarificação mecânica do tegumento, seguida de uma pré-embebição em solução aquosa de ácido giberélico a $1.000 \mathrm{mg} \mathrm{L}^{-1}$ por $72 \mathrm{~h}$; porém, a porcentagem final de germinação foi reduzida devido aos danos causados pela escarificação.

O uso de reguladores de crescimento como giberelinas (BEVILAQUA et al., 1993) e citocininas (CUNHA e CASALI, 1989) na fase de germinação pode melhorar o desempenho de sementes de várias espécies, principalmente sob condições adversas. Frazão e Pinheiro (1981) e Frazão et al. (1981), realizando experimentos com a germinação de amêndoas de palmeira do gênero Orbignya, também observaram maior porcentagem de germinação com ácido giberélico.

Este trabalho objetivou avaliar a eficiência de diferentes tratamentos pré-germinativos para acelerar e uniformizar a germinação das sementes de R. excelsa.

\section{MATERIAL E MÉTODOS}

O experimento foi conduzido na Universidade Federal de Lavras, em Lavras, MG, no Setor de Fisiologia Vegetal do Departamento de Biologia. 
Os frutos foram colhidos em cachos com o mesmo estágio visual de maturação (coloração amarela) na mesma planta-matriz. Os frutos, depois de selecionados e extraídos dos cachos, foram imersos em água para embebição durante três dias. Após esse período, os frutos foram despolpados manualmente, pressionandoos em uma peneira sob água corrente, e as sementes obtidas foram secas à sombra durante $24 \mathrm{~h}$ (AGUIAR et al., 2002). Após a secagem, as sementes foram submetidas aos diferentes tratamentos de escarificação: T1 - lixar um lado da semente (lado do maior comprimento, paralelo ao eixo embrionário); T2 - lixar os dois lados da semente; T3 - imersão em água a $100^{\circ} \mathrm{C}$ por $1 \mathrm{~min}$; T4 - imersão em água a $100^{\circ} \mathrm{C}$ por 2 min; T5 - imersão em água a $100^{\circ} \mathrm{C}$ por 4 min; T6 - imersão em ácido sulfúrico $98 \%$ por $1 \mathrm{~min}$; 77 - imersão em ácido sulfúrico $98 \%$ por 2 min; T8 - imersão em ácido sulfúrico $98 \%$ por 4 min; e T9 - Testemunha. Em seguida, as sementes foram colocadas para germinar em caixas tipo GERBOX, tendo como substrato areia autoclavada (1,1 atm, 123 ${ }^{\circ} \mathrm{C}$ por $45 \mathrm{~min}$ ) a uma umidade de $70 \%$ da capacidade de campo, mantidas em câmara de germinação tipo B.O.D. com fotoperíodo de $12 \mathrm{~h}$ de luz a $30^{\circ} \mathrm{C} \mathrm{e} 12 \mathrm{~h}$ de escuro a $20^{\circ} \mathrm{C}$. O delineamento experimental utilizado foi inteiramente casualizado com nove tratamentos contendo quatro repetições com 25 sementes, totalizando 100 sementes por tratamento.

No segundo ensaio, as sementes foram mergulhadas por $24 \mathrm{~h}$ em soluções contendo BAP (6-Benzilaminopurina) nas concentrações de $0,25,50$ ou $100 \mathrm{mg} \mathrm{L}^{-1}$ e GA (acido giberélico $-\operatorname{Vetec}^{\circledR}$ ) nas concentrações de 0 , 100,200 ou $300 \mathrm{mg} \mathrm{L}^{-1}$, em todas as combinações possíveis, perfazendo um fatorial 4 × 4 . Após os tratamentos, as sementes foram colocadas para germinar em bandejas de isopor (tipo plantagil) contendo como substrato areia autoclavada e mantidas em estufa com sombreamento de $50 \%$ e irrigação por microaspersão. O delineamento experimental utilizado foi um fatorial $4 \times 4$, inteiramente casualizado com 16 tratamentos, contendo quatro repetições com 25 sementes, num total de 100 sementes por tratamento.

Em ambos os ensaios, o índice de velocidade de emergência (IVE) e a porcentagem de emergência foram avaliados semanalmente até que o processo de emergência estivesse estabilizado, quando foi avaliado o número de plântulas normais (plântula cuja plúmula rompia a superfície do substrato), para o cálculo da porcentagem de emergência. OIVE das plântulas foi calculado de acordo com a fórmula proposta por Maguire (1962).
Os resultados observados foram submetidos à análise de variância com o auxílio do programa SISVAR ${ }^{\circledR}$ (FERREIRA, 2000). Utilizou-se o teste de Scott Knott a $5 \%$ de probabilidade, para comparação entre as médias dos tratamentos.

\section{RESULTADOS E DISCUSSÃO}

O uso de escarificação mecânica (T1 e T2) apresentou tendência em aumentar a porcentagem de emergência, porém nãodiferenciou estatisticamente dos tratamentos com a utilização de ácido sulfúrico durante 1 (T6) e 4 min (T8) e a testemunha (T9), conforme mostrado no Quadro 1.

A maior porcentagem de emergência (36\%) foi obtida quando se lixou um lado da semente (T1). Nos tratamentos em que foi utilizada a imersão das sementes em água aquecida a aproximadamente $100^{\circ} \mathrm{C}$ (tratamentos 3 , 4 e 5), não ocorreu germinação. Resultados semelhantes foram obtidos no índice de velocidade de emergência (IVE), conforme se visualiza no Quadro 1.

Kitze (1958), trabalhando com sementes da palmeira Copernicia australis Becc., obteve bons resultados, realizando a escarificação mecânica das sementes. A utilização de ácido sulfúrico também proporcionou bons resultados, porém inferiores se comparado com o uso de escarificação mecânica. Frazão e Pinheiro (1982) também concluíram que o uso de escarificação mecânica estimula e uniformiza a germinação de sementes da palmeira do gênero Orbignya. Ferreira e Gentil (2006), estudando a germinação de tucumã (Astrocaryum aculeatum), confirmaram a possibilidade de se retirar o endocarpo sem causar prejuízos à viabilidade das sementes (germinação superior a 58\%), independentemente dos vários períodos de embebição analisados.

O uso da escarificação térmica (T3, T4, e T5) e a escarificação química (T7) mostraram-se inferiores aos demais tratamentos quanto à porcentagem e velocidade de germinação de sementes (Quadro 1). Resultados semelhantes foram encontrados por Varela et al. (1991), trabalhando com sementes de Samanea saman, as quais foram danificadas e não apresentaram germinação quando submetidas a tratamentos de imersão em água a $100^{\circ} \mathrm{C}$. Berjaketal. (2004) observaramoefeito deletério do tratamento térmico a $100{ }^{\circ} \mathrm{C}$ na germinação de sementes Phoenix reclinata, e os tratamentos de escarificações mecânica e ácida não apresentaram efeito sobre a germinação. Bovi e Cardoso (1976a) verificaram também que a imersão em água quente $\left( \pm 80^{\circ} \mathrm{C}\right)$ prejudicou a germinação de sementes de Euterpe oleraceae.

R. Árvore, Viçosa-MG, v.32, n.5, p.793-798, 2008 
Quadro 1 - Porcentagem de emergência e Índice de Velocidade de Emergência (IVE) de sementes de Rhapis excelsa submetidas a diferentes tratamentos de escarificação. Lavras, MG, 2003

Table 1 - Emergence percentage and Index of Emergence Speed (IES) for Rhapis excelsa seeds, under different scarification treatments. Lavras, MG, 2003

\begin{tabular}{|c|c|c|c|}
\hline Causa da Variação & GL & Emergência $(\%)^{1}$ & IVE \\
\hline Escarificação (E) & 8 & $838,93 * *$ & $0,0054 * *$ \\
\hline$\underline{\text { Resíduo }}$ & 24 & 111,57 & 0,0010 \\
\hline $\mathrm{CV}(\%)$ & & 60,39 & 89,30 \\
\hline \multicolumn{4}{|l|}{ Médias } \\
\hline \multicolumn{4}{|l|}{$\overline{\text { Escarificação }}$} \\
\hline Lixar um lado da semente & & $34,91^{1}(36,00)^{2} \mathrm{a}$ & 0,0948 a \\
\hline Lixar os dois lados da semente & & $33,27(31,00)$ a & 0,0888 a \\
\hline Imersão em água a $100^{\circ} \mathrm{C}$ por 1 minuto & & $0,00(0,00) b$ & $0,0000 \mathrm{~b}$ \\
\hline Imersão em água a $100^{\circ} \mathrm{C}$ por 2 minutos & & $0,00(0,00) b$ & $0,0000 \mathrm{~b}$ \\
\hline Imersão em água a $100^{\circ} \mathrm{C}$ por 4 minutos & & $0,00(0,00) \mathrm{b}$ & $0,0000 \mathrm{~b}$ \\
\hline Imersão em Ac. Sulfúrico $(98 \%)$ por 1 minuto & & $27,31(23,00)$ a & 0,0531 a \\
\hline Imersão em Ac. Sulfúrico (98\%) por 2 minutos & & $13,62(8,00) b$ & $0,0189 \mathrm{~b}$ \\
\hline Imersão em Ac. Sulfúrico (98\%) por 4 minutos & & $22,20(19,00)$ a & $0,0252 \mathrm{~b}$ \\
\hline Testemunha (sem escarificação) & & $26,07(21,00) \mathrm{a}$ & $0,0474 \mathrm{a}$ \\
\hline
\end{tabular}

${ }^{1}$ Dados transformados em arco-seno $(\mathrm{x} / 100)^{1 / 2} \cdot{ }^{2}$ Dados não-transformados. Médias seguidas da mesma letra na coluna não diferem entre si, pelo teste de Skott-Knott a $5 \%$ de probabilidade.

A escarificação térmica inibiu totalmente a germinação, indicando que a exposição das sementes de palmeira-ráfia a temperaturas próximas de $100{ }^{\circ} \mathrm{C}$ pode ter prejudicado a estrutura do embrião.

No experimento em que foram testadas diferentes combinações de doses de $\mathrm{GA}_{3}$ e BAP visando ao estímulo da germinação de sementes de palmeira-ráfia, não houve efeito significativo dos tratamentos aplicados sobre a porcentagem de germinação e o índice de velocidade de emergência (IVE), Quadro 2.

Os resultados variaram de $45 \%$ a $54 \%$ nas porcentagens de emergência, não havendo diferença entre os tratamentos com os reguladores vegetais, embora tenha sido observado, por vários autores como Nagao e Sakai (1979), Nagao et al. (1980), Frazão e Pinheiro (1981) e Frazão et al. (1981), o incremento da germinação com a aplicação de giberelinas em sementes de palmeiras. Scalon et al. (2006a) relataram que não houve efeito da aplicação de giberelina em tratamento pré-germinativo de sementes de jacarandá. Segundo esses autores, as sementes provavelmente possuíam teores endógenos de giberelinas suficientes para a germinação, e a suplementação exógena pode ter causado efeito inibidor. Resultados semelhantes foram observados por Scalon et al. (2006b), estudando a germinação e crescimento inicial da muda de orelha-de-macaco (Enterolobium contortisiliqunn (Vell.) Morong).
Quadro 2-Porcentagem de emergência e Índice de Velocidade de Germinação (IVE) de sementes de Rhapis excelsa submetidas a diferentes doses de reguladores de crescimento. Lavras, MG, 2003

Table 2 - Emergence percentage and Index of Emergence Speed (IVE) for Rhapis excelsa seeds, treated with different amounts of growth regulators. Lavras, $M G$, 2003

\begin{tabular}{|c|c|c|c|}
\hline Causa da Variação & GL & Emergência (\%) ${ }^{1}$ & IVE \\
\hline Citocinina & 3 & $64,64^{\mathrm{NS}}$ & $0,0015^{\mathrm{NS}}$ \\
\hline $\mathrm{GA}_{3}$ & 3 & $46,73^{\mathrm{NS}}$ & $0,0009^{\mathrm{NS}}$ \\
\hline Interação & 9 & $63,05^{\mathrm{NS}}$ & $0,0016^{\mathrm{NS}}$ \\
\hline \multicolumn{4}{|l|}{ Citocinina $\mathrm{x} \mathrm{GA}_{3}$} \\
\hline Resíduo & 45 & 69,80 & 0,0010 \\
\hline$\overline{\mathrm{CV}(\%)}$ & & 18,58 & 27,71 \\
\hline \multicolumn{4}{|l|}{ Médias } \\
\hline \multicolumn{4}{|l|}{ Citocinina } \\
\hline $0 \mathrm{mg} \mathrm{L}^{-1}$ & & $42,24^{1}(45,50)^{2} \mathrm{a}$ & $0,1047 \mathrm{a}$ \\
\hline $25 \mathrm{mg} \mathrm{L}^{-1}$ & & $47,05(54,00) \mathrm{a}$ & $0,1247 \mathrm{a}$ \\
\hline $50 \mathrm{mg} \mathrm{L}^{-1}$ & & $45,00(50,00)$ a & $0,1211 \mathrm{a}$ \\
\hline $100 \mathrm{mg} \mathrm{L}^{-1}$ & & $45,56(51,00)$ a & $0,1259 \mathrm{a}$ \\
\hline \multicolumn{4}{|l|}{$\overline{\mathrm{GA}_{3}}$} \\
\hline $0 \mathrm{mg} \mathrm{L}^{-1}$ & & $47,52(54,50) \mathrm{a}$ & $0,1301 \mathrm{a}$ \\
\hline $100 \mathrm{mg} \mathrm{L}^{-1}$ & & $44,30(49,25) \mathrm{a}$ & $0,1141 \mathrm{a}$ \\
\hline $200 \mathrm{mg} \mathrm{L}^{-1}$ & & $43,94(48,25)$ a & $0,1136 \mathrm{a}$ \\
\hline $300 \mathrm{mg} \mathrm{L}^{-1}$ & & $44,10(48,50) \mathrm{a}$ & $0,1186 \mathrm{a}$ \\
\hline
\end{tabular}


O aumento nos valores porcentuais de germinação por ação das giberelinas também já foi relatado em vários trabalhos com diferentes espécies de frutíferas (DUARTE, 1982). Segundo Metivier (1986), giberelinas e citocininas estão envolvidas na quebra da dormência de sementes de diferentes espécies, causando diminuição na velocidade média de germinação, sendo esse fato muito importante, pois o tempo médio de germinação é considerado como a primeira etapa para a redução no tempo de formação da muda. Os tratamentos prégerminativos de sementes da palmeira-ráfia, utilizandose os reguladores de crescimento $\mathrm{GA}_{3}$ e BAP, não influenciaram a porcentagem de germinação e o índice de velocidade de emergência das sementes da espécie.

\section{CONCLUSÃO}

Pelos resultados do ensaio deste trabalho, podese concluir que na germinação de sementes de palmeiraráfia não é recomendado o uso de escarificação ou da aplicação de reguladores de crescimento para acelerar esse processo.

\section{REFERÊNCIAS}

AGUIAR, F. F. A. et al. Germinação de sementes de Rhapis excelsa (Thunb.) Henry ex Rehder: efeitos da temperatura, luz e substrato.

Hoehnea, v.32, n.1, p.119-26, 2005.

AGUiAR, F. F. A. et al. Produção de mudas de palmito-juçara Euterpe edulis Mart. São Paulo: Instituto de Botânica, 2002. 16p.

BERJAK, P.; von FINTEL, G. T.; PAMMENTER, N. W. Seed behaviour in Phoenix reclinata Jacquin, the wild date palm. Seed Science Research, v.14, n.2, p.197-204, 2004.

BEVILAQUA, G. A. P. et al. Desempenho de sementes de arroz irrigado tratadas com regulador de crescimento. I. Efeito na emergência a campo.

Revista Brasileira de Sementes, v.15, n.1, p.75-80, 1993.

BOVI, M. L. A., CARDOSO, M. Germinação de sementes de açaizeiro I. Bragantia, v.35, n.1, p.50-56, 1976a.

BOVI, M. L. A., CARDOSO, M. Germinação de sementes de palmiteiro II. Bragantia, v.35, n.1, p.23-29, $1976 b$.
BROSCHAT, T. K. Palm seed propagation. Acta Horticulture, v.360, p.141-147, 1994.

CARVAlho, N. M.; NAKAGAWA, J. Sementes, ciência, tecnologia e produção. 3.ed. Campinas: Fundação Cargil, 1988. 424p.

CUNHA, R.; CASALI, V. W. Efeito de substâncias reguladoras do crescimento sobre a germinação de sementes de alface. Revista Brasileira de Fisiologia Vegetal, v.1, n.2, p.121-132, 1989.

DOMINGUES, R. C. Espécies ornamentais. Natureza, v.5, n.8, p.14-18, 1995.

DUARTE, O. Propagation methods for tropical and subtropical fruits. Proceedings of the International Horticultural Congress, v.1, n.21, p.415-424, 1982.

ELIAS, M. E. A.; FERREIRA, S. A. N.; GENTIL, D. F.O. Emergência de plântulas de tucumã (Astrocaryum aculeatum) em função da posição de semeadura. Acta Amazonica, v.36, n.3, p.385-388, 2006.

FERREIRA, D. F. Análise estatística por meio do SISVAR (Sistema para Análise de Variância) para Windows versão 4.0. In: REUNIÃO ANUAL DA REGIÃO BRASILEIRA DA SOCIEDADE INTERNACIONAL DE BIOMETRIA, 45., 2000, São Carlos. Anais... São Carlos: Universidade Federal de São Carlos, 2000. p.255-258.

FERREIRA, S. A. N.; GENTIL, D. F.O. Extração, embebição e germinação de sementes de tucumã (Astrocaryum aculeatum). Acta Amazonica, v.36, n.2, p.141-146, 2006.

FIGLIOLIA, M. B.; YAMAZOE, G., SILVA, A. Germinação de sementes de Euterpe edulis Mart. em condições de laboratório e viveiro após tratamentos pré-germinativos. Boletim do Instituto Florestal, v.41, n.2, p.343-353, 1987.

FRAZÃO, F. M. F.; PINHEIRO, C. U. B. Experimentos com germinação de amêndoas de babaçu (Orbignya spp.) II. São Luiz: Inst. Est. Babaçu, 1981. (Manuscrito).

FRAZÃO, F. M. F.; PINHEIRO, C. U. B. Implantação do banco ativo de germoplasma de babaçu (Orbignya spp.). São Luiz: Inst. Est. Babaçu, 1982. (Relatório Técnico).

R. Árvore, Viçosa-MG, v.32, n.5, p.793-798, 2008 
FRAZÃO, J. M. F.; PINHEIRO, C. U. B.; KURY, N. S. Experimentos com germinação de amêndoas de babaçu (Orbignya spp.) - I. São Luiz: Inst. Est. Babaçu, 1981. (Manuscrito)

KITZE, E. D. A method for germinating Copernicia palm seeds. Principes, v.2, n.1, p.5-8, 1958.

KOEBERNICK, J. Germination of palm seed. Principes, v.15, n.2, p.134-137, 1971.

LORENZI, H. et al. Palmeiras brasileiras e exóticas cultivadas. Nova Odessa: Plantarum, 2004. 390p.

MACIEL, N. M. S. Efectos de la madurez y el almacenamiento del fruto, la escarificacion y el remojo de las semillas sobre la emergencia de la palma china de abanico. Agronomía Tropical, v.46, n.2, p.155-170, 1996.

MAEDA, J. A. et al. Germinação de sementes da palmeira Archontophoenix alexandrae. In: ENCONTRO NACIONAL SOBREARBORIZAÇÃO URBANA, 1987, Maringá. Anais... Maringá: PMM/SBAU, 1987. p.99-107.

MAGUIRE, J. D. Speed of germination aid in selection and evaluation for seedling emergence and vigor. Crop Science, v.2, n.2, p.176-177, 1962.

MARTINS, C. C. et al. Despolpamento e temperatura no armazenamento temporário de sementes de palmito-vermelho (Euterpe espiritosantensis Fernandes). Revista Brasileira de Sementes, v.22, n.1, p.169-176, 2000.

McKAMEY, L. Rhapis Palms - Culitivated Species \& Varieties Culture and Care of the "Ladies". Principes, v.33, n.3, p.129-139, 1989.

MEEROW, A. W. Palm seed germination. Fort Lauderdale: IFAS Cooperative Extension Service, 1991. 10p. (Bulletin, 274).

METIVIER, J. R. Citocininas e giberilinas. In: FERRI, M. G. Fisiologia vegetal. 2.ed. São Paulo: EDUSP, 1986. v.2. p.93-162.

MULLETT, T. H.; BEARDSELL, D. V.; KING, H. M. The effect of seed treatment on the germination and early growth of Euterpe edulis (Family Palmae). Scientia Horticulturae, v.15, n.3, p.239-244, 1981.

NAGAO, M. A.; KANEGAWA, K.; SAKAI, W. S. Accelerating palm seed germination with gibberelic acid scarification and bottom heat. Horticultural Science, v.15, n.2, p.200-201, 1980.

R. Árvore, Viçosa-MG, v.32, n.5, p.793-798, 2008
NAGAO, M. A.; SAKAI, W. S. Effect of growth regulators on seed germination of Archontophoenix alexandrae. HortScience, v.14, n.2, p.182-183, 1979.

ODETOLA, J. A. Studies on seed dormancy, viability, and germination in ornamental palms. Principes, v.31, n.1, p.24-30, 1987.

PIVETTA, K. F. L. et al. Effects of maturation and scarification on seed germination of Syagrus schizophylla (Mart.) Glass. (Arecaceae). Acta Horticulturae, v.683, p.375-378, 2005.

POPINIGIS, F. Fisiologia da semente. 2.ed. Brasília: ABEAS, 1985. 289p.

SCALON, S. P. Q. et al. Armazenamento e tratamento pré-germinativos em sementes de jacarandá (Jacaranda cuspidifolia Mart.).

Revista Árvore, v.30, n.2, p.179-185, 2006 a.

SCALON, S. P. Q. et al. Germinação e crescimento inicial da muda de orelha-de-macaco (Enterolobium contortisiliqunn (Vell.) Morong): efeito de tratamentos químicos e luminosidade. Revista Árvore, v.30, n.4, p.529-536, 2006 b.

VARELA, V. P.; BROCKI, E.; SÁ, S. T. V. Tratamentos pré-germinativos de espécies da Amazônica IV. Faveira camuzê - Stryphnodendron pulcherimum (Willd.) Hochr - Leguminosae.

Revista Brasileira de Sementes, v.13, n.2, p.87-89, 1991.

VIANA, F. A. P. Estudos sobre germinação e morfo-anatomia do diásporo e da plântula de Livistona rotundifolia (Lam.) Mart. (Arecaceae). 2003. 76f. Dissertação (Mestrado em Produção e Tecnologia de Sementes) - Universidade Estadual Paulista, Jaboticabal, 2003.

VILLALOBOS, R.; HERRERA, J.; GUEVARA, E. Germinacion de la semilla de pejibaye (Bactris gasipaes). II. Ruptura Del reposo. Agronomia Costarricense, v.16, n.1, p.61-68, 1992a.

VILLALOBOS, R.; HERRERA, J.; MORA-URPI, J. Germinacion de la semilla de pejibaye (Bactris gasipaes). III. Efecto del contenido de agua y de las condiciones de almacenamiento. Agronomia Costarricense, v.16, n.1, p.69-76, 1992b. 